\title{
An examination of dietary intakes and nutritional status of chronic healthy spinal cord injured individuals
}

\author{
Alan M Levine PhD RD, ${ }^{1 *}$ Mark S Nash PhD,${ }^{2}$ Barth A Green MD,${ }^{2} \mathbf{J}$ Darrell Shea \\ $\mathrm{MD},{ }^{3}$ Michael J Aronica $\mathrm{MD}^{4}$ \\ ${ }^{1}$ Marywood College, Scranton, PA; ${ }^{2}$ University of Miami School of Medicine, Miami, \\ FL; ${ }^{3}$ Lucerne Rehabilitation and Spinal Center, Humana Hospital-Lucerne, Orlando, FL; \\ ${ }^{4}$ Allied Services Institute of Rehabilitation, Scranton, PA, USA.
}

To examine the nutritional composition of the dietary intake of chronic healthy spinal cord injured (SCI) individuals, 33 subjects affiliated with 3 SCI rehabilitation centers logged their food consumption for 7 days. Prior to record keeping, subjects were trained by a registered dietitian on the accurate recording of their standard food choices and portion size, and were provided with scales to weigh food accurately. Dietary macro and micronutrients were analyzed with a computer software package, with nutritional analysis compared to the recommended daily allowances (RDA) of the Food and Nutrition Board of the National Academy of Sciences. Analysis showed caloric intake to be $75 \%$ of that recommended for able bodied persons, with a high reliance on fat for calories. Fat intake accounted for $37.9 \%$ of calories, well above the recommended level of $30 \%$, but typical of the American diet. The ratio of polyunsaturated to saturated fat was approximately one half the recommended level, with carbohydrate calories averaging $16.5 \%$ below optimal RDA. Protein consumption was within normal range, but average dietary fiber was only $25 \%$ of recommended levels. Micronutrient analysis showed deficiencies in both water and fat-soluble vitamins, with suboptimal intake of multiple minerals. Given the apparent reliance on a high-fat and low-carbohydrate diet, this research shows that nutritional intervention and education of SCI persons are needed, and that a registered dietitian should be included in the SCI health care team.

Keywords: spinal cord injury; diet; nutrition.

\section{Introduction}

In recent years, nutrition and dietary management of spinal cord injured (SCI) patients have received meaningful attention in the literature. Researchers have examined nutritional status during the acute ${ }^{1-3}$ as well as chronic phase of recovery ${ }^{4-11}$ following SCI. Given, however, that limited information exists concerning longitudinal nutrient intake of SCI individuals, that previous studies have been performed on relatively small sample populations, and that dietary habits change with time, updated research documenting the nutritional status of SCI

*Correspondence: Department of Human Ecology, Marywood College, Scranton, Pennsylvania 18509, USA. individuals is of importance to the SCI health care professional. This information may be utilized to anticipate dietary deficiencies, assist in the design of optimal nutritional programs, and prevent sequelae associated with diets that are either deficient of excessive in essential nutrients. The purpose of this study was to compare the dietary intakes and nutritional status of SCI persons with those of both the able bodied population and commonly accepted guidelines for optimal dietary management.

\section{Methods}

Subjects for the study were 33 chronic healthy SCI patients ( 24 male, 9 female) affiliated with 3 SCI rehabilitation centers 
(University of Miami/Jackson Memorial Medical Center, Miami, Florida; Lucerne Rehabilitation and Spinal Center, Humana Hospital-Lucerne, Orlando, Florida; and Allied Services Institute of Rehabilitation, Scranton, Pennsylvania). Demographic data were collected for each subject including age, gender, income level, educational level, living arrangements, person(s) responsible for meal preparation, level of injury, duration of injury, and frequency and duration of physical activity.

A 7-day dietary record and a food frequency chart were used to assess the nutrient intake of subjects. A food scale, pen, notebook and intake forms were given to all subjects by the researchers. The forms contained columns for recording the time and location of food consumption, a description of the food and/or beverage consumed, and the amount consumed. Subjects were instructed by a registered dietitian (RD) on correct procedures for recording portion sizes, with guidelines for weighing, measuring and recording foods given both orally and in writing.

Initially, all subjects completed and returned a 2-day sample dietary record. These data were reviewed by the RD for compliance with the oral and written instructions and immediately returned to study participants. Additional training was provided if needed. Subjects were then instructed to record their food and drink consumption for 7 consecutive days. At the end of that period all records were returned to the project representative at each study center.

The nutrient content of the 7-day dietary recall logs was analyzed with a nutritional software package, the Food Processor II. ${ }^{12}$ Descriptive statistics were calculated from the analysis, and the dietary intakes compared to the 1989 recommended dietary allowances (RDA) ${ }^{13}$ or other authoritative goals/recommendations. ${ }^{14-16}$

\section{Results}

The sample consisted of 11 paraplegics and 21 quadriplegics (one individual did not reply to the level of injury question). The majority of subjects were aged between 20 and 30 years, had annual incomes below $\$ 20,000$, and had not completed a 4-year college degree. At the time of the study most individuals had been injured for longer than 4 years, lived with their families, and had help with meal preparation. The individuals in the sample population were quite active, with 19 individuals performing at least some exercise activity each day. On a weekly basis, 22 individuals reported activity for 1 hour or more (Table I).

Nutrient intakes for the subjects are reported in Table II. These data were differentiated by gender to allow for direct comparison with the 1989 RDA guidelines, which are gender-specific. Table III provides a comparison of subject nutrient intake with the RDA guidelines or other authoritative goals and recommendations. Analysis of the mean data for the study participants suggest that the male subjects had intakes of vitamin $\mathrm{A}$, thiamin, riboflavin, pyridoxine, vitamin E, calcium, magnesium and zinc that were below the RDA. Intakes of potassium, pantothenate and copper were below the lower end of the suggested safe range of the National Research Council (NRC). Female subjects had deficiencies of pyridoxine, calcium, iron, magnesium and zinc intake, and reported intakes of copper below the lower end of the aforementioned NRC range. In fact, many subject (males and females) showed intakes below the so-called 'safety level' of two thirds of the RDA.

With respect to caloric intake from the 3 nutrient groups, fat consumption for males and females were $7.6 \%$ and $1.5 \%$ above the $30 \%$ American Heart Association (AHA) guidelines, respectively. Carbohydrate intakes for males and females were $9.3 \%$ and $4.7 \%$ below the lower end of the $55-60 \%$ AHA recommendations, respectively, while protein intakes were $1.7 \%$ and $2 \%$ above the upper end of the $10-15 \%$ AHA suggestions. As was found with many of the study variables, these ranges and standard deviations suggest wide variation of dietary composition among study participants.

Although cholesterol intakes were below the $300 \mathrm{mg}$ AHA guidelines, the ratios of polyunsaturated to saturated fat (P:S) of 0.5 
Table I Demographic information on the study subjects $(n=33)^{*}$

\begin{tabular}{|c|c|c|c|}
\hline Variables & Categories & $n$ & $\%$ \\
\hline Age & $\begin{array}{l}10-20 \text { years } \\
21-30 \text { years } \\
31-40 \text { years } \\
41-50 \text { years } \\
\text { Over } 50 \text { years }\end{array}$ & $\begin{array}{r}2 \\
17 \\
7 \\
6 \\
1\end{array}$ & $\begin{array}{r}6.1 \\
51.2 \\
21.2 \\
18.2 \\
3.0\end{array}$ \\
\hline Sex & $\begin{array}{l}\text { Male } \\
\text { Female }\end{array}$ & $\begin{array}{r}24 \\
9\end{array}$ & $\begin{array}{l}72.7 \\
27.3\end{array}$ \\
\hline Income & $\begin{array}{l}\text { Under } \$ 10,000 \\
\$ 10,000-\$ 20,000 \\
\$ 20,000-\$ 30,000 \\
\$ 30,000-\$ 40,000 \\
\text { Over } \$ 40,000\end{array}$ & $\begin{array}{r}12 \\
5 \\
5 \\
2 \\
8\end{array}$ & $\begin{array}{r}37.5 \\
15.6 \\
15.6 \\
6.3 \\
25.0\end{array}$ \\
\hline Education & $\begin{array}{l}\text { HS degree/equivalency } \\
\text { Some college } \\
\text { College degree } \\
\text { Post baccalaureate }\end{array}$ & $\begin{array}{r}16 \\
4 \\
7 \\
5\end{array}$ & $\begin{array}{l}50.0 \\
12.5 \\
21.9 \\
15.6\end{array}$ \\
\hline Living arrangements & $\begin{array}{l}\text { Lives alone } \\
\text { Lives with family } \\
\text { Group situation }\end{array}$ & $\begin{array}{r}6 \\
23 \\
4\end{array}$ & $\begin{array}{l}18.2 \\
69.7 \\
12.1\end{array}$ \\
\hline Meal preparation & $\begin{array}{l}\text { Self } \\
\text { Family member } \\
\text { Friend }\end{array}$ & $\begin{array}{r}8 \\
20 \\
5\end{array}$ & $\begin{array}{l}24.2 \\
60.6 \\
15.2\end{array}$ \\
\hline Level of injury & $\begin{array}{l}\text { Paraplegic } \\
\text { Quadraplegic }\end{array}$ & $\begin{array}{l}11 \\
21\end{array}$ & $\begin{array}{l}34.4 \\
65.6\end{array}$ \\
\hline Time of injury & $\begin{array}{l}1-2 \text { years ago } \\
2-3 \text { years ago } \\
3-4 \text { years ago } \\
\text { Over } 4 \text { years ago }\end{array}$ & $\begin{array}{r}7 \\
7 \\
3 \\
16\end{array}$ & $\begin{array}{r}21.2 \\
21.2 \\
9.1 \\
48.5\end{array}$ \\
\hline Frequency of activity & $\begin{array}{l}1-2 \text { times/week } \\
3-4 \text { times/week } \\
5-6 \text { times/week } \\
7 \text { or more times/week }\end{array}$ & $\begin{array}{r}3 \\
7 \\
1 \\
19\end{array}$ & $\begin{array}{r}10.0 \\
23.3 \\
3.3 \\
63.3\end{array}$ \\
\hline Length of activity & $\begin{array}{l}\text { Under } 15 \mathrm{~min} / \text { week } \\
15-29 \mathrm{~min} / \text { week } \\
30-44 \mathrm{~min} / \text { week } \\
45-59 \mathrm{~min} \\
1 \text { hour or more/week }\end{array}$ & $\begin{array}{r}3 \\
2 \\
0 \\
4 \\
22\end{array}$ & $\begin{array}{c}9.7 \\
6.5 \\
0 \\
12.9 \\
71.0\end{array}$ \\
\hline
\end{tabular}

*Some individuals did not answer all demographic questions.

$\mathrm{HS}=$ high school .

for males and 0.64 for females were well below the 1.0 AHA target. Additionally, consumption of fiber for both males and females of 12.2 and $14.3 \mathrm{~g}$, respectively, was well below the National Cancer Institute (NCI) guideline of $20-30 \mathrm{~g} /$ day. ${ }^{15}$

Table IV allows for comparison of study participant intakes to the mean intake of nutrients by the US population as a whole.
Food consumption data from a number of surveys, as reported by the National Research Council/National Academy of Science, ${ }^{13,16}$ suggest similarities of protein intake for study participants and the general US population. Carbohydrate intake provided an average of $45.3 \%$ and $46.4 \%$ of total energy intake for men and women, respectively. Thus, females in the present 
Table II Mean nutrient intake during 7-day period $(n=33)$

\begin{tabular}{|c|c|c|c|c|c|}
\hline \multirow{2}{*}{$\begin{array}{l}\text { Nutrient } \\
\text { Energy } \\
\text { (Kcal) }\end{array}$} & \multirow[b]{2}{*}{$\begin{array}{l}(\mathrm{M}) \\
(\mathrm{F})\end{array}$} & \multirow{2}{*}{ 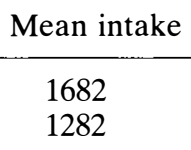 } & \multirow{2}{*}{\multicolumn{2}{|c|}{$\begin{array}{c}\text { Range } \\
2486-847\end{array}$}} & \multirow{2}{*}{$\frac{\mathrm{SD}}{429.1}$} \\
\hline & & & & & \\
\hline $\begin{array}{l}\text { Protein } \\
\text { (g) }\end{array}$ & $\begin{array}{l}(\mathrm{M}) \\
(\mathrm{F})\end{array}$ & $\begin{array}{l}69 \\
56\end{array}$ & $\begin{array}{r}122 \\
84\end{array}$ & $\begin{array}{l}-\quad 30 \\
-\quad 25\end{array}$ & $\begin{array}{l}20.7 \\
21.5\end{array}$ \\
\hline $\begin{array}{l}\text { Carbohydrates } \\
\text { (g) }\end{array}$ & $\begin{array}{l}(\mathrm{M}) \\
(\mathrm{F})\end{array}$ & $\begin{array}{l}204 \\
166\end{array}$ & $\begin{array}{l}460- \\
235\end{array}$ & $\begin{array}{l}-86 \\
-\quad 83\end{array}$ & $\begin{array}{l}81.0 \\
52.2\end{array}$ \\
\hline $\begin{array}{l}\text { Fiber } \\
(\mathrm{g})\end{array}$ & $\begin{array}{l}(\mathrm{M}) \\
(\mathrm{F})\end{array}$ & $\begin{array}{l}12.2 \\
14.3\end{array}$ & $\begin{array}{l}22.7 \\
27.6\end{array}$ & $\begin{array}{l}-\quad 6.0 \\
-\quad 4.4\end{array}$ & $\begin{array}{l}4.7 \\
8.8\end{array}$ \\
\hline $\begin{array}{l}\text { Fat } \\
(\mathrm{g})\end{array}$ & $\begin{array}{l}(\mathrm{M}) \\
(\mathrm{F})\end{array}$ & $\begin{array}{l}67 \\
47\end{array}$ & $\begin{array}{r}124 \\
87\end{array}$ & $\begin{array}{l}-\quad 30.3 \\
-\quad 15.8\end{array}$ & $\begin{array}{l}23.9 \\
22.6\end{array}$ \\
\hline $\begin{array}{l}\text { Saturated fat } \\
(\mathrm{g})\end{array}$ & $\begin{array}{l}(\mathrm{M}) \\
(\mathrm{F})\end{array}$ & $\begin{array}{l}25 \\
16\end{array}$ & $\begin{array}{l}45.5 \\
28.0\end{array}$ & $\begin{array}{l}12.7 \\
-\quad 5.3\end{array}$ & $\begin{array}{l}9.0 \\
7.2\end{array}$ \\
\hline $\begin{array}{l}\text { Monounsaturated fat } \\
\text { (g) }\end{array}$ & $\begin{array}{l}(\mathrm{M}) \\
(\mathrm{F})\end{array}$ & $\begin{array}{l}25 \\
17\end{array}$ & $\begin{array}{l}51.4 \\
29.1\end{array}$ & $\begin{array}{lr}- & 11.1 \\
- & 5.5\end{array}$ & $\begin{array}{l}9.8 \\
7.5\end{array}$ \\
\hline $\begin{array}{l}\text { Polyunsaturated fat } \\
\text { (g) }\end{array}$ & $\begin{array}{l}(\mathrm{M}) \\
(\mathrm{F})\end{array}$ & $\begin{array}{l}12 \\
11\end{array}$ & $\begin{array}{l}24.8 \\
24.7\end{array}$ & $\begin{array}{l}-\quad 3.6 \\
-\quad 3.8\end{array}$ & $\begin{array}{l}5.8 \\
7.0\end{array}$ \\
\hline $\begin{array}{l}\text { Cholesterol } \\
(\mathrm{mg})\end{array}$ & $\begin{array}{l}(\mathrm{M}) \\
(\mathrm{F})\end{array}$ & $\begin{array}{l}246 \\
200\end{array}$ & $\begin{array}{l}521 \\
391\end{array}$ & $\begin{array}{l}-126 \\
-\quad 83\end{array}$ & $\begin{array}{r}96.8 \\
102.0\end{array}$ \\
\hline $\begin{array}{l}\text { Vitamin A } \\
\text { (RE) }\end{array}$ & $\begin{array}{l}(\mathrm{M}) \\
(\mathrm{F})\end{array}$ & $\begin{array}{l}815 \\
929\end{array}$ & $\begin{array}{l}1946 \\
2437\end{array}$ & $\begin{array}{l}-153 \\
-196\end{array}$ & $\begin{array}{l}455.3 \\
824.4\end{array}$ \\
\hline $\begin{array}{l}\text { Thiamin } \\
(\mathrm{mg})\end{array}$ & $\begin{array}{l}(\mathrm{M}) \\
(\mathrm{F})\end{array}$ & $\begin{array}{l}1.32 \\
1.37\end{array}$ & $\begin{array}{l}2.29 \\
2.99\end{array}$ & $\begin{array}{ll}9- & 0.729 \\
9- & 0.42\end{array}$ & $\begin{array}{l}0.43 \\
0.79\end{array}$ \\
\hline $\begin{array}{l}\text { Riboflavin } \\
(\mathrm{mg})\end{array}$ & $\begin{array}{l}(\mathrm{M}) \\
(\mathrm{F})\end{array}$ & $\begin{array}{l}1.46 \\
1.50\end{array}$ & $\begin{array}{l}2.56 \\
3.48\end{array}$ & $\begin{array}{ll}6- & 0.81 \\
8- & 0.52\end{array}$ & $\begin{array}{l}0.45 \\
1.03\end{array}$ \\
\hline $\begin{array}{l}\text { Niacin } \\
(\mathrm{mg})\end{array}$ & $\begin{array}{l}(\mathrm{M}) \\
(\mathrm{F})\end{array}$ & $\begin{array}{l}21.2 \\
20.0\end{array}$ & $\begin{array}{l}31.8 \\
39.4\end{array}$ & $\begin{array}{lc}- & 11.1 \\
- & 6.03\end{array}$ & $\begin{array}{r}5.78 \\
12.52\end{array}$ \\
\hline $\begin{array}{l}\text { Pyridoxine } \\
\text { (mg) }\end{array}$ & $\begin{array}{l}(\mathrm{M}) \\
(\mathrm{F})\end{array}$ & $\begin{array}{l}1.51 \\
1.57\end{array}$ & $\begin{array}{l}2.62 \\
3.78\end{array}$ & $\begin{array}{ll}52- & 0.69 \\
8- & 0.51\end{array}$ & $\begin{array}{l}0.49 \\
1.11\end{array}$ \\
\hline $\begin{array}{l}\text { Vitamin } B_{12} \\
(\mathrm{mg})\end{array}$ & $\begin{array}{l}(\mathrm{M}) \\
(\mathrm{F})\end{array}$ & $\begin{array}{l}4.32 \\
4.30\end{array}$ & $\begin{array}{l}21.7 \\
11.0\end{array}$ & $\begin{array}{l}-\quad 0.26 \\
-\quad 0.64\end{array}$ & $\begin{array}{l}4.03 \\
3.47\end{array}$ \\
\hline $\begin{array}{l}\text { Folacin } \\
(\mathrm{mg})\end{array}$ & $\begin{array}{l}(\mathrm{M}) \\
(\mathrm{F})\end{array}$ & $\begin{array}{l}215.6 \\
219.1\end{array}$ & $\begin{array}{l}422 \\
583\end{array}$ & $\begin{array}{l}-\quad 91 \\
-\quad 68\end{array}$ & $\begin{array}{r}84.3 \\
166.2\end{array}$ \\
\hline $\begin{array}{l}\text { Pantothenate } \\
\text { (mg) }\end{array}$ & $\begin{array}{l}(\mathrm{M}) \\
(\mathrm{F})\end{array}$ & $\begin{array}{l}3.65 \\
4.75\end{array}$ & $\begin{array}{c}9.61- \\
13.7\end{array}$ & $\begin{array}{l}51- \\
5.12 \\
-\quad 1.46\end{array}$ & $\begin{array}{l}1.53 \\
3.80\end{array}$ \\
\hline $\begin{array}{l}\text { Vitamin C } \\
(\mathrm{mg})\end{array}$ & $\begin{array}{l}(\mathrm{M}) \\
(\mathrm{F})\end{array}$ & $\begin{array}{r}103 \\
81\end{array}$ & $\begin{array}{l}312 \\
142\end{array}$ & $\begin{array}{l}-\quad 19 \\
-\quad 26\end{array}$ & $\begin{array}{l}81.3 \\
41.8\end{array}$ \\
\hline $\begin{array}{l}\text { Vitamin E } \\
(\mathrm{mg} \mathrm{TE})\end{array}$ & $\begin{array}{l}(\mathrm{M}) \\
(\mathrm{F})\end{array}$ & $\begin{array}{r}7.8 \\
11.1\end{array}$ & $\begin{array}{l}17.9 \\
24.9\end{array}$ & $\begin{array}{l}-\quad 1.68 \\
-\quad 3.25\end{array}$ & $\begin{array}{l}4.3 \\
7.3\end{array}$ \\
\hline $\begin{array}{l}\text { Calcium } \\
(\mathrm{mg})\end{array}$ & $\begin{array}{l}(\mathrm{M}) \\
(\mathrm{F})\end{array}$ & $\begin{array}{l}550 \\
525\end{array}$ & $\begin{array}{r}1549 \\
965\end{array}$ & $\begin{array}{l}-245 \\
-205\end{array}$ & $\begin{array}{l}268.3 \\
262.9\end{array}$ \\
\hline $\begin{array}{l}\text { Copper } \\
(\mathrm{mg})\end{array}$ & $\begin{array}{l}(\mathrm{M}) \\
(\mathrm{F})\end{array}$ & $\begin{array}{l}1.1 \\
1.1\end{array}$ & $\begin{array}{l}1.67- \\
1.85\end{array}$ & $\begin{array}{ll}57- & 0.64 \\
35- & 0.48\end{array}$ & $\begin{array}{l}0.30 \\
0.46\end{array}$ \\
\hline $\begin{array}{l}\text { Iron } \\
(\mathrm{mg})\end{array}$ & $\begin{array}{l}(\mathrm{M}) \\
(\mathrm{F})\end{array}$ & $\begin{array}{l}12.0 \\
13.5\end{array}$ & $\begin{array}{l}22.0 \\
32.5\end{array}$ & $\begin{array}{l}-6.66 \\
-\quad 6.14\end{array}$ & $\begin{array}{l}3.98 \\
8.33\end{array}$ \\
\hline $\begin{array}{l}\text { Magnesium } \\
(\mathrm{mg})\end{array}$ & $\begin{array}{l}(\mathrm{M}) \\
(\mathrm{F})\end{array}$ & $\begin{array}{l}217 \\
242\end{array}$ & $\begin{array}{l}380 \\
443\end{array}$ & $\begin{array}{l}-135 \\
-\quad 85\end{array}$ & $\begin{array}{r}65.1 \\
123.2\end{array}$ \\
\hline $\begin{array}{l}\text { Phosphorus } \\
\text { (mg) }\end{array}$ & $\begin{array}{l}(\mathrm{M}) \\
(\mathrm{F})\end{array}$ & $\begin{array}{r}1005 \\
915\end{array}$ & $\begin{array}{r}487 \\
1721\end{array}$ & $\begin{array}{l}-441 \\
-400\end{array}$ & $\begin{array}{l}304.5 \\
419.1\end{array}$ \\
\hline
\end{tabular}


Table II (cont)

\begin{tabular}{|c|c|c|c|c|c|}
\hline Nutrient & & Mean intake & & Range & SD \\
\hline $\begin{array}{l}\text { Potassium } \\
(\mathrm{mg})\end{array}$ & $\begin{array}{l}(\mathrm{M}) \\
(\mathrm{F})\end{array}$ & $\begin{array}{l}1975 \\
1828\end{array}$ & $\begin{array}{l}3313 \\
3144\end{array}$ & $\begin{array}{l}-972 \\
-697\end{array}$ & $\begin{array}{l}540.9 \\
762.8\end{array}$ \\
\hline $\begin{array}{l}\text { Selenium } \\
(\mathrm{mg})\end{array}$ & $\begin{array}{l}(\mathrm{M}) \\
(\mathrm{F})\end{array}$ & $\begin{array}{l}98 \\
86\end{array}$ & $\begin{array}{l}169 \\
152\end{array}$ & $\begin{array}{l}-\quad 43 \\
-\quad 27\end{array}$ & $\begin{array}{l}28.2 \\
40.5\end{array}$ \\
\hline $\begin{array}{l}\text { Sodium } \\
(\mathrm{mg})\end{array}$ & $\begin{array}{l}(\mathrm{M}) \\
(\mathrm{F})\end{array}$ & $\begin{array}{l}2594 \\
1949\end{array}$ & $\begin{array}{l}3983 \\
2990\end{array}$ & $\begin{array}{l}-1037 \\
-1139\end{array}$ & $\begin{array}{l}690.1 \\
649.6\end{array}$ \\
\hline $\begin{array}{l}\text { Zinc } \\
(\mathrm{mg})\end{array}$ & $\begin{array}{l}(\mathrm{M}) \\
(\mathrm{F})\end{array}$ & $\begin{array}{r}10.2 \\
9.3\end{array}$ & $\begin{array}{l}18.7 \\
22.7\end{array}$ & $\begin{array}{l}7-\quad 3.7 \\
7-\quad 3.7\end{array}$ & $\begin{array}{l}4.11 \\
5.77\end{array}$ \\
\hline $\begin{array}{l}\% \text { Energy from } \\
\text { protein }\end{array}$ & $\begin{array}{l}(\mathrm{M}) \\
(\mathrm{F})\end{array}$ & $\begin{array}{l}16.7 \\
17\end{array}$ & $\begin{array}{l}26 \\
21\end{array}$ & $\begin{array}{l}-\quad 9 \\
-\quad 10\end{array}$ & $\begin{array}{l}4.25 \\
3.12\end{array}$ \\
\hline $\begin{array}{l}\% \text { Energy from } \\
\text { carbohydrate }\end{array}$ & $\begin{array}{l}(\mathrm{M}) \\
(\mathrm{F})\end{array}$ & $\begin{array}{l}45.7 \\
51.3\end{array}$ & $\begin{array}{l}65 \\
65\end{array}$ & $\begin{array}{l}-\quad 24 \\
-\quad 36\end{array}$ & $\begin{array}{r}10.33 \\
9.29\end{array}$ \\
\hline $\begin{array}{l}\% \text { Energy from } \\
\text { fat }\end{array}$ & $\begin{array}{l}(\mathrm{M}) \\
(\mathrm{F})\end{array}$ & $\begin{array}{l}37.6 \\
31.5\end{array}$ & $\begin{array}{l}50 \\
47\end{array}$ & $\begin{array}{l}-\quad 24 \\
-\quad 22\end{array}$ & $\begin{array}{l}7.97 \\
8.11\end{array}$ \\
\hline $\mathrm{P}: \mathrm{S}$ & $\begin{array}{l}(\mathrm{M}) \\
(\mathrm{F})\end{array}$ & $\begin{array}{l}0.5 \\
0.64\end{array}$ & $\begin{array}{l}1.0 \\
0.9\end{array}$ & $\begin{array}{l}0-0.2 \\
9-\quad 0.3\end{array}$ & $\begin{array}{l}0.23 \\
0.22\end{array}$ \\
\hline
\end{tabular}

$\mathrm{M}$ = male

$\mathrm{F}=$ female

$\mathrm{RE}=$ retinol equivalents

$\mathrm{TE}=$ tocopherol equivalents

$\mathrm{P}: \mathrm{S}=$ ratio of polyunsaturated to saturated fat.

study appear to meet the dietary recommendations more closely than do females in the general population. Typical fat content of the US diet was found to be $36-37 \%$ of total calories. Males in the present study consumed similar amounts, but females were approximately $5 \%$ lower than the general population. Cholesterol intakes for both genders of SCI subjects were appreciably lower than those of the general population.

\section{Discussion}

The literature of spinal cord injury has grown dramatically since the pioneering work of Sir Ludwik Guttmann, with treatments focusing on acute stabilization, surgical management, and numerous aspects of rehabilitation care filling the pages of many SCI topical journals and books. Given the vast body of knowledge accumulated over the past decade concerning dietary influences on the wellbeing of both healthy and diseased individuals, it is ironic that only a handful of these articles reflect analysis of, or concern about, the dietary intake and nutritional status of paralyzed individuals. That diets deficient or excessive in nutrients may be associated with multiple sequelae commonly linked with SCI, including delayed wound healing, cardiovascular risk, immunodeficiency and body composition, has been addressed previously, ${ }^{7}$ research that illustrates the importance of investigating the influence of dietary intake and nutrient metabolism upon the medical management and health of the paralyzed individual. It is acknowledged that no single nutritional variable or study design can adequately reflect the global nutritional status of any individual. Additionally, the data collected in this study reflect the oral intake of nutrients and not an analysis of their relative uptake, metabolism or excretion. Nevertheless, the study results suggest that both nutritional strengths and weaknesses are to be found in the food choices of SCI persons.

With reference to the 3 major dietary nutrients, the results of the study demonstrate that dietary protein levels of the study 
Table III Comparison between mean nutrient intake of SCI subjects and the 1989 RDA (or other authoritative goal/recommendation)

\begin{tabular}{|c|c|c|c|c|}
\hline \multirow{2}{*}{$\begin{array}{l}\text { Nutrient } \\
\text { Protein } \\
(\mathrm{g})\end{array}$} & \multicolumn{3}{|r|}{ RDA } & \multirow{2}{*}{$\begin{array}{c}\text { Intake as } \% \text { of RDS } \\
110 \\
112\end{array}$} \\
\hline & $\begin{array}{l}(\mathrm{M}) \\
(\mathrm{F})\end{array}$ & $\begin{array}{l}63^{\mathrm{a}} \\
50\end{array}$ & & \\
\hline $\begin{array}{l}\text { Fiber } \\
(\mathrm{g})\end{array}$ & $\begin{array}{l}(\mathrm{M}) \\
(\mathrm{F})\end{array}$ & $\begin{array}{l}20 \\
20\end{array}$ & $\begin{array}{l}-30^{\mathrm{b}} \\
-30\end{array}$ & $\begin{array}{l}61^{\mathrm{d}} \\
71\end{array}$ \\
\hline Saturated fat & $\begin{array}{l}(\mathrm{M}) \\
(\mathrm{F})\end{array}$ & $\begin{array}{l}10 \%^{c} \\
10 \%\end{array}$ & & $\begin{array}{l}13^{\mathrm{f}} \\
11\end{array}$ \\
\hline Monounsaturated fat & $\begin{array}{l}(\mathrm{M}) \\
(\mathrm{F})\end{array}$ & $\begin{array}{l}10 \%^{c} \\
10 \%\end{array}$ & & $\begin{array}{l}13^{\mathrm{f}} \\
12\end{array}$ \\
\hline Polyunsaturated fat & $\begin{array}{l}(\mathrm{M}) \\
(\mathrm{F})\end{array}$ & $\begin{array}{l}10 \%{ }^{c} \\
10 \%\end{array}$ & & $\begin{array}{l}6^{\mathrm{f}} \\
8\end{array}$ \\
\hline $\begin{array}{l}\text { Cholesterol } \\
(\mathrm{mg})\end{array}$ & $\begin{array}{l}(\mathrm{M}) \\
(\mathrm{F})\end{array}$ & $\begin{array}{l}300^{c} \\
300\end{array}$ & & $\begin{array}{l}82 \\
67\end{array}$ \\
\hline $\begin{array}{l}\text { Vitamin A } \\
(\mathrm{RE})\end{array}$ & $\begin{array}{l}(\mathrm{M}) \\
(\mathrm{F})\end{array}$ & $\begin{array}{c}1000^{\mathrm{a}} \\
800\end{array}$ & & $\begin{array}{r}82 \\
116\end{array}$ \\
\hline $\begin{array}{l}\text { Thiamin } \\
(\mathrm{mg})\end{array}$ & $\begin{array}{l}(\mathrm{M}) \\
(\mathrm{F})\end{array}$ & $\begin{array}{l}1.5^{\mathrm{a}} \\
1.1\end{array}$ & & $\begin{array}{r}88 \\
125\end{array}$ \\
\hline $\begin{array}{l}\text { Riboflavin } \\
(\mathrm{mg})\end{array}$ & $\begin{array}{l}(\mathrm{M}) \\
(\mathrm{F})\end{array}$ & $\begin{array}{l}1.7^{\mathrm{a}} \\
1.3\end{array}$ & & $\begin{array}{r}86 \\
115\end{array}$ \\
\hline $\begin{array}{l}\text { Niacin } \\
(\mathrm{mg})\end{array}$ & $\begin{array}{c}(\mathrm{M}) \\
(\mathrm{F})\end{array}$ & $\begin{array}{l}19^{\mathrm{a}} \\
15\end{array}$ & & $\begin{array}{l}111 \\
134\end{array}$ \\
\hline $\begin{array}{l}\text { Pyridoxine }\left(\mathrm{B}_{6}\right) \\
(\mathrm{mg})\end{array}$ & $\begin{array}{c}(\mathrm{M}) \\
(\mathrm{F})\end{array}$ & $\begin{array}{l}2.0^{\mathrm{a}} \\
1.6\end{array}$ & & $\begin{array}{l}76 \\
98\end{array}$ \\
\hline $\begin{array}{l}\text { Vitamin } B_{12} \\
(\mu \mathrm{g})\end{array}$ & $\begin{array}{l}(\mathrm{M}) \\
(\mathrm{F})\end{array}$ & $\begin{array}{l}2.0^{\mathrm{a}} \\
2.0\end{array}$ & & $\begin{array}{l}216 \\
215\end{array}$ \\
\hline $\begin{array}{l}\text { Folacin } \\
(\mu \mathrm{g})\end{array}$ & $\begin{array}{l}(\mathrm{M}) \\
(\mathrm{F})\end{array}$ & $\begin{array}{l}200^{\mathrm{a}} \\
180\end{array}$ & & $\begin{array}{l}108 \\
122\end{array}$ \\
\hline $\begin{array}{l}\text { Pantothenate } \\
(\mathrm{mg})\end{array}$ & $\begin{array}{l}(\mathrm{M}) \\
(\mathrm{F})\end{array}$ & $\begin{array}{l}4 \\
4\end{array}$ & $\begin{array}{l}-\quad 7^{a} \\
-\quad 7\end{array}$ & $\begin{array}{c}91^{\mathrm{d}} \\
119\end{array}$ \\
\hline $\begin{array}{l}\text { Vitamin C } \\
(\mathrm{mg})\end{array}$ & $\begin{array}{l}(\mathrm{M}) \\
(\mathrm{F})\end{array}$ & $\begin{array}{l}60^{\mathrm{a}} \\
60\end{array}$ & & $\begin{array}{l}172 \\
135\end{array}$ \\
\hline $\begin{array}{l}\text { Vitamin E } \\
\text { (TE) }\end{array}$ & $\begin{array}{l}(\mathrm{M}) \\
(\mathrm{F})\end{array}$ & $\begin{array}{c}10^{\mathrm{a}} \\
8\end{array}$ & & $\begin{array}{r}78 \\
111\end{array}$ \\
\hline $\begin{array}{l}\text { Calcium } \\
(\mathrm{mg})\end{array}$ & $\begin{array}{l}(\mathrm{M}) \\
(\mathrm{F})\end{array}$ & $\begin{array}{l}800^{a} \\
800\end{array}$ & & $\begin{array}{l}69 \\
66\end{array}$ \\
\hline $\begin{array}{l}\text { Copper } \\
(\mathrm{mg})\end{array}$ & $\begin{array}{l}\text { (M) } \\
\text { (F) }\end{array}$ & $\begin{array}{l}1.5 \\
1.5\end{array}$ & $\begin{array}{l}-\quad 3.0^{\mathrm{a}} \\
-\quad 3.0\end{array}$ & $\begin{array}{l}73^{d} \\
73\end{array}$ \\
\hline $\begin{array}{l}\text { Iron } \\
(\mathrm{mg})\end{array}$ & $\begin{array}{c}(\mathrm{M}) \\
(\mathrm{F})\end{array}$ & $\begin{array}{l}10^{\mathrm{a}} \\
15\end{array}$ & & $\begin{array}{r}120 \\
90\end{array}$ \\
\hline $\begin{array}{l}\text { Magnesium } \\
(\mathrm{mg})\end{array}$ & $\begin{array}{c}(\mathrm{M}) \\
(\mathrm{F})\end{array}$ & $\begin{array}{l}350^{\mathrm{a}} \\
280\end{array}$ & & $\begin{array}{l}62 \\
86\end{array}$ \\
\hline $\begin{array}{l}\text { Phosphorus } \\
\text { (mg) }\end{array}$ & $\begin{array}{c}(\mathrm{M}) \\
(\mathrm{F})\end{array}$ & $\begin{array}{l}800^{\mathrm{a}} \\
800\end{array}$ & & $\begin{array}{l}126 \\
114\end{array}$ \\
\hline $\begin{array}{l}\text { Potassium } \\
(\mathrm{mg})\end{array}$ & $\begin{array}{c}(\mathrm{M}) \\
(\mathrm{F})\end{array}$ & $\begin{array}{l}2000 \\
2000\end{array}$ & $\begin{array}{l}-3500^{c} \\
-3500\end{array}$ & $\begin{array}{l}99^{\mathrm{d}} \\
91\end{array}$ \\
\hline $\begin{array}{l}\text { Selenium } \\
(\mu \mathrm{g})\end{array}$ & $\begin{array}{l}(\mathrm{M}) \\
(\mathrm{F})\end{array}$ & $\begin{array}{l}70^{\mathrm{a}} \\
55\end{array}$ & & $\begin{array}{l}140 \\
156\end{array}$ \\
\hline
\end{tabular}


Table III (cont)

\begin{tabular}{|c|c|c|c|}
\hline Nutrient & & RDA & Intake as $\%$ of RDS \\
\hline $\begin{array}{l}\text { Sodium } \\
\text { (mg) }\end{array}$ & $\begin{array}{l}\text { (M) } \\
\text { (F) }\end{array}$ & $\begin{array}{l}2400^{c} \\
2400\end{array}$ & $\begin{array}{r}108 \\
81\end{array}$ \\
\hline $\begin{array}{l}\text { Zinc } \\
(\mathrm{mg})\end{array}$ & $\begin{array}{l}(\mathrm{M}) \\
(\mathrm{F})\end{array}$ & $\begin{array}{l}15^{\mathrm{a}} \\
12\end{array}$ & $\begin{array}{l}68 \\
78\end{array}$ \\
\hline $\begin{array}{l}\% \text { Energy from } \\
\text { protein }\end{array}$ & $\begin{array}{l}(\mathrm{M}) \\
(\mathrm{F})\end{array}$ & $\begin{array}{l}-15^{c} \\
-\quad 15\end{array}$ & $\begin{array}{l}16.7 \\
17\end{array}$ \\
\hline $\begin{array}{c}\text { \% Energy from } \\
\text { carbohydrate }\end{array}$ & $\begin{array}{l}(\mathrm{M}) \\
(\mathrm{F})\end{array}$ & $\begin{array}{l}-60^{c} \\
-60\end{array}$ & $\begin{array}{l}45.7 \\
51.3\end{array}$ \\
\hline $\begin{array}{l}\% \text { Energy from } \\
\text { fat }\end{array}$ & $\begin{array}{l}\text { (M) } \\
\text { (F) }\end{array}$ & $\begin{array}{l}30^{c} \\
30\end{array}$ & $\begin{array}{l}37.6 \\
31.5\end{array}$ \\
\hline $\mathrm{P}: \mathrm{S}$ & $\begin{array}{l}\text { (M) } \\
\text { (F) }\end{array}$ & $\begin{array}{l}1.0^{c, f} \\
1.0\end{array}$ & $\begin{array}{l}0.5^{\mathrm{f}} \\
0.64\end{array}$ \\
\hline
\end{tabular}

a Reference 13

${ }^{\circ}$ Reference 15

${ }^{\text {c Reference } 14}$

${ }^{\mathrm{d}}$ Comparison was made to lower end of range

${ }^{\text {e }}$ Reference 16

${ }^{\mathrm{f}}$ Actual intake, not calculated as a percentage

$\mathrm{M}=$ male

$\mathrm{F}=$ female

$\mathrm{RE}=$ retinol equivalents

$\mathrm{TE}=$ tocopherol equivalents

$\mathrm{P}: \mathrm{S}=$ ratio of polyunsaturated to saturated fat.

subjects actually exceed the RDA, suggesting that a state of relative positive nitrogen balance potentially exists in the subjects. In contrast, dietary intake of fat by SCI subjects was higher than recommended levels, and carbohydrate consumption was limited, both of which are cause for concern. SCI persons are known to be at increased risk for cardiovascular disease secondary to a relatively sedentary lifestyle. ${ }^{17}$ The reported death rates of SCI persons due to coronary artery disease are more than double those of age- and gender-matched able bodied persons,${ }^{18}$ with cardiopulmonary complications accounting for $40.5 \%$ of all known causes of SCI-related death. ${ }^{19}$ While it is recognized that many factors unrelated to diet contribute to cardiopulmonary morbidity and mortality in SCI persons, it is also understood that an atherosclerotic diet may contribute, in part, to exacerbation or acceleration of cardiopulmonary disease in individuals so predisposed by their paralysis.

The finding of restricted intake of dietary carbohydrate by the study subjects has consequences for suboptimal sugar, protein and fatty acid metabolism both at rest and during physical activity. While the main function of carbohydrate is to serve as an energy fuel for muscle contraction, at rest, it also serves to spare protein for tissue maintenance, repair and growth. During exercise it functions as a primer for fatty acid catabolism by providing the oxaloacetate intermediate in the Krebs' cycle essential for catabolism of acetyl-CoA monomers derived from beta-oxidation of fatty acids. While the increased level of physical activity of the study subjects is highly desirable, their restricted intake of carbohydrate does not facilitate use of fatty acids as a fuel source during subaximal steady-state exercise, and may actually dispose them to muscle glycogen depletion and exerciseinduced ketosis during either high-intensity of long-duration physical activity. Because more SCI persons are involving themselves in physical activities incuding wheelchair sports and therapies, ${ }^{20}$ the consumption of dietary carbohydrate may serve as a limiting 
Table IV Nutrient intake of US population

\begin{tabular}{l} 
Nutrient \\
\hline$\%$ Energy from \\
protein \\
$\%$ Energy from \\
fat \\
$\%$ Energy from \\
carbohydrate
\end{tabular}

Fiber

(g)

Protein

(g)

Fat

(g)

Carbohydrate

(g)

Saturated fat

(g)

Monounsaturated fat

(g)

Polyunsaturated fat

(g)

\section{Cholesterol}

(mg)

Vitamin A

(RE)

Thiamin

(mg)

Riboflavin

(mg)

Niacin

(mg)

Pyridoxine

(mg)

Folacin

$(\mu \mathrm{g})$

Vitamin $\mathrm{B}_{12}$

$(\mu \mathrm{g})$

Pantothenate

(mg)

Vitamin C

(mg)

Vitamin E

(TE)

Calcium

(mg)

Phosphorus

(mg)

Magnesium

(mg)
Intake

$16.5^{\mathrm{a}}$

(M)

(F)

(M)

(F)

(M)

(F)

(M)

(F)

(M)

(F)

(M)

(F) $\quad 36.8^{\mathrm{a}, \mathrm{c}}$

(M) $\quad 287^{\mathrm{b}}$

(F) $\quad 177$

(M/F) $\quad 13.3^{\mathrm{a}, \mathrm{c}}$

$(\mathrm{M} / \mathrm{F}) \quad 13.6^{\mathrm{a} . \mathrm{c}}$

$(\mathrm{M} / \mathrm{F}) \quad 7.4^{\mathrm{a} . \mathrm{c}}$

(M) $\quad 439^{\mathrm{a}}$

(F) 280

(M) $1419^{\mathrm{b}}$

(F) 1170

(M) $\quad 1.75^{\mathrm{b}}$

(F) $\quad 1.05$

(M) $\quad 2.08^{\mathrm{b}}$

(F) $\quad 1.39$

(M) $\quad 41^{\mathrm{b}}$

(F) 27

(M) $\quad 1.87 \mathrm{~b}$

(F) $\quad 1.16$

(M) $\quad 305^{\mathrm{a}}$

(F) 189

(M) $\quad 7.84^{\mathrm{b}}$

(F) $\quad 4.85$

$(\mathrm{M} / \mathrm{F}) \quad 6^{\mathrm{b}}$

(M) $109^{\mathrm{b}}$

(F) 77

(M) $\quad 9.8^{\mathrm{b}}$

(F) $\quad 7.1$

(M) $\quad 920^{\mathrm{a}}$

(F) 592

(M) $1500^{\mathrm{b}}$

(F) 1000

(M) $329^{\mathrm{b}}$

(F) 207
Table IV (cont)

\begin{tabular}{lcc}
\hline Nutrient & & Intake \\
\hline $\begin{array}{l}\text { Iron } \\
(\mathrm{mg})\end{array}$ & $(\mathrm{M})$ & $15.9^{\mathrm{a}}$ \\
$\begin{array}{l}\text { Zinc } \\
(\mathrm{mg})\end{array}$ & $(\mathrm{F})$ & 10.1 \\
$\begin{array}{l}\text { Selenium } \\
(\mu \mathrm{g})\end{array}$ & $8.4^{\mathrm{a}}$ \\
$\begin{array}{l}\text { Copper } \\
(\mathrm{mg})\end{array}$ & $(\mathrm{M} / \mathrm{F})$ & $108^{\mathrm{a}}$ \\
$\begin{array}{l}\text { Sodium } \\
(\mathrm{mg})\end{array}$ & $(\mathrm{M})$ & $1.6^{\mathrm{a}}$ \\
$\begin{array}{l}\text { Potassium } \\
(\mathrm{mg})\end{array}$ & $(\mathrm{F})$ & 1.0 \\
\hline & $(\mathrm{M})$ & $1569 / 1000 \mathrm{kcal}^{\mathrm{a}}$ \\
\hline
\end{tabular}

a Reference 16

bReference 13

${ }^{c}$ Data is reported as a percentage and not the absolute value

$\mathrm{M}=$ male

$\mathrm{F}=$ female

$\mathrm{RE}=$ retinol equivalents

$\mathrm{TE}=$ tocopherol equivalents.

factor in attaining their fullest rehabilitative, recreational or athletic potential.

While it is generally agreed that fiber is not a nutrient, dietary fiber has received considerable research attention because of the established relationship between highfiber diet and lowered incidence of obesity, intestinal disorders and heart disease. ${ }^{21}$ Analysis of dietary fiber of SCI males showed that intake was approximately one third less than the mean intake of the general population. However, SCI females consumed almost $40 \%$ more than their female counterparts in the larger population. Increased amounts of dietary fiber for SCI persons may be necessary to enhance neurogenic bowel function, as well as prevent various fiber-linked disorders. It has been suggested that fiber may assist in lowering of serum cholesterol in humans and may favorably alter the ratios of high- to low-density lipoproteins towards a less atherogenic blood profile. ${ }^{22,23}$ The precise mechanisms by which fiber alters blood lipoprotein concentrations are still unclear, but may be related to hindrance of cholesterol absorption in the gut, depressed synthesis of cholesterol or excretion of existing 
cholesterol bound to fiber in the feces. ${ }^{24}$ Heightened resistance to either hypercholesterolemia or heart disease in SCI individuals might be realized with intakes closer to the NCI guidelines. ${ }^{25}$ The data is indicative of an ongoing concern for some of the atherosclerotic risk factors.

That some of the nutrient assessments suggested that SCI subjects do not differ from their able bodied counterparts is hardly comforting. The diet of the average American is commonly accepted to be too high in fat and refined sugar, and too restricted in complex carbohydrate. Further, it must be considered that the consequences of poor diet, including difficulty in maintaining optimal body weight, may profoundly affect various aspects of a paralytic's life, including the daily activities of wheelchair or brace locomotion and transfers, and may also influence their perception of body image and the ability to participate in recreational or sporting activities. Additionally, body fat once acquired may be more difficult to shed by the paralyzed person due to the limited muscle mass available to increase metabolism when compared to able bodied persons.

\section{Conclusions}

We conclude that food intake of SCI persons differs in many respects both from optimal dietary standards and from the intake of the general population. Intakes of most vitamins and minerals for both SCI males and females were below those of the general population. The percentage of calories from the 3 energy nutrients suggests a need for dietary modification, including slightly lower protein, increased complex carbohydrate and decreased fat intakes. The elevated levels of dietary fat in the diets of SCI subjects coupled with low levels of dietary fiber, their disposition to cardiovascular disease, and (in some cases) nominal activity levels place them at heightened risk for cardiopulmonary morbidity and mortality already accepted as common for persons sustaining SCI. As the study results indicate that SCI patients are at some nutritional risk, we conclude that an important role may present for the dietitian/nutritionist as part of the allied health care team that treats and counsels paraplegics and quadriplegics on aspects of nutrition. The establishment of optimal dietary requirements for the SCI individuals based upon their unique physiology and scientific analysis of their metabolic needs requires additional study.

\section{Acknowledgement}

The assistance of Judy White, Allied Services Institute of Rehabilitation, Scranton, PA, for dietary analysis is gratefully acknowledged.

\section{References}

1 Dietz JM, Bertschy M, Gschaedler R, Dollfus P (1986) Reflections on the intensive care of 106 acute cervical spinal cord injury patients in the resuscitation unit of a general traumatology centre. Paraplegia 24(6): 343 .

2 Huang CT, DeVivo MJ, Stover SL (1990) Anemia in acute phase of spinal cord injury. Arch Phys Med Rehab 71(1): 3 .

3 Laven GT, Huang CT, DeVivo MJ, Stover SL, Kuhlemeier KV, Fine PR (1989) Nutritional status during the acute stage of spinal cord injury. Arch Phys Med Rehab 70(4): 277.

4 Barboriak JJ, Rooney CB, El Ghatit AZ, Spude K Anderson AJ (1983) Nutrition in spinal cord injury patients. J Am Para Soc 6(2): 32.

5 Gines DJ, Holliday ME, Ervin K (1987) Nutrition assessment during rehabilitation of the spinal cord injury patient. Top Clin Nutr 2(4): 39.

6 Lagger L (1983) Spinal cord injury: nutritional management. J Neurosurg Nurs 15(5): 310.

7 Lee BY, Agarwal N, Corcoran L, Thoden WR, DelGuercio LR (1985) Assessment of nutritional and metabolic status of paraplegics. J Rehab Res Dev 22(3): 11.

8 Mitchell E (1984) Nutritional care plan for spinal cord injured patients. Spin Cord Inj Nurs 1(2): 13.

9 Peiffer SC, Blust P, Leyson JF (1981) Nutritional assessment of the spinal cord injured patient. JADA 78(5): 501

10 Perkash A, Brown M (1986) Anemia in patients with traumatic spinal cord injury. J Am Para Soc 9(1-2): 10.

11 Shizgal HM, Roza A, Leduc B, Drouin G, Villemure JG, Yaffe C (1986) Body composition in quadriplegic patients. J Parenter Enteral Nutr 10(4): 364. 
12 ESHA Research (1987) The Food Processor II. Salem, Oregon.

13 Food and Nutrition Board, National Research Council (1989) Recommended Dietary Allowances. 10th rev. ed. National Academy Press, Washington, DC.

14 American Heart Association (1988) Dietary guidelines for healthy American adults. Circulation 77: 721A.

15 Butrum RR, Clifford CK, Lanza E (1988) National Cancer Institute guidelines: rationale. Am J Clin Nutr 48 (Suppl): 888.

16 Committee on Diet and Health, Food and Nutrition Board and Committee on Life Sciences, National Research Council (1989) Diet and Health: Implications for Reducing Chronic Disease Risk. National Academy Press, Washington DC.

17 LaPorte RE, Adams LL, Savage DD, Brenes G, Dearwater S, Cook T (1984) The spectrum of physical activity, cardiovascular disease and health: and epidemiologic perspective. J Epidem 120(4): 507.

18 Le CT, Price M (1982) Survival from spinal cord injury. Chronic Dis 35: 487-492.

19 DeVivo MJ (1988) Life expectancy and causes of death. In: The National Consensus Conference on Catastrophic Illness and Injury. The Spinal Cord Injury Model: Lessons Learned and New Applications. Sponsored by the National Institutes of Disability and Rehabilitation Research.

20 Glaser RM (1985) Exercise and locomotion for the spinal cord injured. Ex and Sports Sci Rev 13: 263.

21 Kay RM (1982) Dietary fiber. J Lipid Res 23: 221.

22 Kirby RW, Anderson JW, Sieling B, Rees ED, Chen WL, Miller RE, et al (1981) Oat-bran intake selectively lowers serum low-density lipoprotein cholesterol concentrations in hypercholesterolemic men. Am J Clin Nutr 34: 824.

23 Superko HR, Haskell WL, Sawrey-Kubicek L, Farquhar JW (1988) The effect of solid and liquid gum on the reduction of plasma cholesterol in patients with moderate hypercholesterolemia. Am J Cardiol 62: 51.

24 Eastwood MA, Kay RM (1979) An hypothesis for the action of dietary fiber along the gastrointestinal tract. Am J Clin Nutr 32: 364.

25 Kromhout D, Bosschieter EB, Coulander CD (1982) Dietary fiber and 10 year mortality from coronary heart disease, cancer, and all causes. Lancet ii: 518. 